\title{
Perseveration in Tool Use: A Window for Understanding the Dynamics of the Action-Selection Process
}

\author{
Ad W. Smitsman and Ralf F. A. Cox \\ Behavioral Science Institute, \\ Radboud University Nijemgen
}

\begin{abstract}
Two experiments investigated how 3-year-old children select a tool to perform a manual task, with a focus on their perseverative parameter choices for the various relationships involved in handling a tool: the actor-to-tool relation and the tool-to-target relation (topology). The first study concerned the parameter value for the tool-to-target relation by asking how children use a cane for either pushing an object further away (exclosure; outside the hook) or pulling an object nearby (enclosure; inside the hook). The second study concerned the parameter value for the hand-to-tool relation by assessing the hand used for grasping a spoon to feed a puppet. Results from both studies showed that on the first trial, choices were driven by task information. However, when the task switched from pulling to pushing or from left hand to right hand, or vice versa, children persevered with the choice they made during the 4 previous trials. Results are discussed in terms of the dynamical field modeling work of Esther Thelen and her colleagues. Our findings underscore Thelen's hypotheses that: (a) the action-selection process is a dynamic affair, affected by multiple influences at different time scales and its own intrinsic dynamics; and (b) that perseverative behavior is a general phenomenon that is neither indicative for a specific period in the development, nor for a particular task. Similarly, we argue that the presence (or absence) of causal understanding emerges from the action-selection process, rather than determining this process.
\end{abstract}

The planning and control of goal-directed behavior entails many action choices. Some of them are made after conscious deliberation, many of them made uncon-

Correspondence should be sent to Ad W. Smitsman, Radboud University Nijmegen, Behavioral Science Institute, Department of Developmental Psychology, P.O. Box 9104, 6500 HE Nijmegen, The Netherlands. E-mail: a.smitsman@psych.ru.nl 
sciously and automatically. However, in all these choices a dynamic choice process underlies the decisions that are made over time. Choices are needed because of the variety of goals that can be attained and the many degrees of freedom of the body and the environment that need to be constrained to attain a goal. As a general framework, choices have to be made with respect to goals, means, and the specific parameter settings that interconnect goals and means. Goals, broadly speaking, refer to potential end states for an organism and its environment. Means refer to body segments, often complemented by environmental objects (i.e., bodily attachments or tools), and organizations of these segments and objects, which the organism can mobilize to accomplish desired end states. Parameter settings refer to the specific values that the relevant behavioral variables need to get in the sequencing of activities that transform the initial state of the organism-environment system into the end state (i.e., the goal). They constrain the degrees of freedom of this system in such a way that potential means transform into effective goal-related devices (see, e.g., Goldfield, 1995; Smitsman, Cox, \& Bongers, 2005).

\section{ACTION SELECTION AND MEANS-END INSIGHT IN TOOL USE}

The ability to select goals that agree with available means and the related ability to select means appropriate to goals form the cornerstone of skilled action. In addition, selecting correct parameter setting(s) not only ensures that action is effective, but also that it is efficient and fluent in its execution. Therefore, to understand how skillful action unfolds and develops and, in particular, how young children develop insight into how to act, it is important to study their action selection. The research report here addresses this issue in young children, in tasks that entail the use of a hand-held tool. More specifically, it addresses the parameters children set for the tool-to-target relation (i.e., hooked stick to object; Experiment 1), and the actor-to-tool relation (i.e., hand to spoon; Experiment 2). Both relationships form key elements in the total system of child, tool, and target. These relationships connect the body with the tool and the tool with the target, and accordingly transform a potential tool object into an effective means to an end (Smitsman et al., 2005). The actor-to-tool relation concerns the way the tool is (to be) grasped and held. The tool-to-target relation concerns the way the object is (to be) touched by the tool, which allows for transportation.

Studying young children's action selection with respect to the two preceding relations is interesting for several reasons. Tool use forms an important developmental milestone, and develops at the beginning of the second year of life, when children mobilize their newly acquired cognitive skills and motor skills to explore object affordances for manipulation of object-object events (Lockman, 2000, 2005; Smitsman, 1997). This also marks the development of additional cognitive 
skills, such as problem solving and imitation, and reveals their understanding of physical objects and physical events (for a recent overview and discussion see Bushnell, Sidman, \& Brugger, 2005). These skills and insights come together in what developmentalists have called means-ends insight. Study of the action-selection process in tool use provides a window for what children's means-ends insight entails, how it develops, and the processes that drive this development.

What are the underlying processes that bring about the means-end insight? What are the driving forces that give rise to children's insight of what to do and what to do next when they explore and start to use objects as tools? In the past, cognitive theorists have attributed a lack of means-end insight in young children to various deficits, and consequently its emergence in older children to various (cognitive) competences that replace previous shortages. Among these competences are physical knowledge of objects (Baillargeon, 1994; Tomasello, 1999; Willats, 1999), representational skills (Piaget, 1954), attention (Boudreau \& Bushnell, 2000; Bushnell, 1985), and the inability to resolve conflicts between goals and subgoals (Baillargeon, 1998; Willats, 1999). On the other hand, action-oriented developmental psychologists have pointed to children's growing manual skill and dexterity (Gibson \& Pick, 2000; Lockman, 2000; Smitsman, 1997; Smitsman \& Bongers, 2003). They have used Gibson's (1979/1986) affordance concept as a theoretical starting point, and have suggested that these skills lead to the discovery of new affordances of objects that involve the various opportunities that handheld objects provide for their use as tools. This position underscores the continuity of tool use in the development of children's action skills as presented earlier (Lockman, 2000; Smitsman, 1997; Smitsman \& Bongers, 2003).

\section{ESTHER THELEN'S WORK ON ACTION PLANNING AND PERSEVERATION}

The dynamical system approach of Esther Thelen and her collegues (e.g., Schöner \& Thelen, 2006; Thelen, Schöner, Scheier, \& Smith, 2001; Thelen \& Smith, 1994; Thelen \& Whitmeyer, 2005) is foundational for this study. It offers an exiting new perspective for studying the development of means-end insight and tool use. Thelen's work on action planning challenges us to pay attention to the action-selection process, its dynamics, and the multiple influences at different time scales. This enables us to better understand children's action choices for a particular task and the child's emerging means-end insight for that task. Studying action selection may also reveal why at one time children demonstrate understanding of the task demands, pay attention to the relevant properties of a task, or perceive the affordances of an object, whereas at other times all of these seem to fail them. This reveals itself when they choose an inappropriate object, an inappropriate way to 
use the object, or sequence the activities inappropriately. (Even in skilled performers one can occasionally observe such failures.) Studying children's action selection will give us new insight into how such differences in behavior can arise on different occasions.

It might be argued that means-end insight results from the confluence of several embodied processes that are active during task performance (Schöner \& Thelen, 2006; Thelen et al., 2001; see also Cox \& Smitsman, 2006a, 2006b). Some of these processes have their origin in the perception-action coupling that arises from performing the task; others go back to earlier experiences with this and similar tasks and the perceptual and motor preferences that result from those experiences. When discussing the physical knowledge one attributes to infants in so called violation of expectancy tasks, Thelen and Whitmeyer (2005) concluded, "Our take-home message is that knowledge of objects is created by the particular spatial and temporal properties of the task, and that concepts do not exist independently of those tasks" (p. 248). In relation to means-end insight this suggests that this insight is a function of informational variables about the task and events, as well as action variables, including preferences and past experiences. Together, these variables influence the decision about how to act (see Schöner \& Thelen, 2006 , for a more elaborate discussion of this issue). Consequently, study of the action-selection process and what influences this process in more detail opens a window for understanding how this determines the presence or absence of means-end insight.

To illustrate the action-selection process, consider the famous A-not-B object permanence task as studied by Thelen and colleagues (Schöner \& Dineva, 2007; Thelen et al., 2001). In this task, children have to decide about the movement parameter for retrieving an object that was previously hidden at one of two locations. The task poses a problem for 8- to 12-month-old infants; after they have successfully retrieved the object at one location, $A$, in a series of trials, they fail to solve the same problem when they have to switch to location $B$, to retrieve the object. Infants of 8 to 12 months persist in reaching toward A. This peculiar phenomenon depends, among other things, on the time delay between placement of the object at B by the experimenter and the child's reaching attempt, and the number of successful retrievals at $\mathrm{A}$. For example, some infants make spontaneous errors by alternating between both locations on the first series of A trials. These infants persevere less compared to infants who do not alternate. The work of Thelen et al. (2001) fosters an interesting view on perseveration. Fundamentally, their work gives rise to the idea that the same process that is responsible for the "incorrect" (i.e., perseverative) reaches is also responsible for the "correct" reaches. They used a dynamical field model to explicate how various, simultaneously present and time-varying influences coact so that correct reaching behavior can emerge in older children, and incorrect reaching in younger children. 


\section{RELATION WITH TOOL USE}

Although reaching for a location in space, as in the A-not-B task, seems a simpler and different task than choosing a tool and deciding how to use it, they are similar in fundamental ways. In both tasks, choices have to be made between different alternatives that are simultaneously present. Moreover, in both tasks, varying influences coact over time and affect the action-selection process. Because these influences are not static but vary over time, along different time scales, it follows that action selection itself is dynamic. It has been suggested before that these aspects of the process are more general, and thus not limited to the A-not-B task, and neither related to a specific period in young children's development. The approach and modeling technique is not content specific, which means that it may be applied to a huge variety of tasks (e.g., Schöner \& Dineva, 2007). It turned out that this enabled precise modeling of infants' looking behavior in so called violation-of-expectancy tasks (Schöner \& Thelen, 2006). Moreover, Schutte and Spencer (2002; see also Schutte, Spencer, \& Schöner, 2003) showed similar perseverative reaching in older children. To take this further, Thelen's work provides us with new and refreshing insights and methods for studying the action-selection process in tool use.

There are also empirical and task-analytical arguments that demand us to pay more attention to the action-selection process when studying means-ends insight in, for example, tool use. Several studies have documented the observation that young children's means-ends insight is highly influenced by task factors (Bates, Carlson-Luden, \& Bretherton, 1980; Bushnell \& Boudreau, 1998; Cox \& Smitsman, 2006b; Van Leeuwen, Smitsman, \& Van Leeuwen, 1994). For instance, Van Leeuwen et al. (1994) showed that young children's willingness to use a cane to retrieve an attractive object, which is placed beyond reach on a table, depended on the way the task was set up. In their setup, they placed the cane within reach and in proximity of the child in various orientations and distances to the object. Several, mostly younger, children used the cane when a simple pulling or sliding movement was sufficient to enclose the object in the hook of the cane. However, they refused to use it when they had to rotate the cane first, before sliding or pulling made enclosure of the cookie possible. Recently, Cox and Smitsman (2006b) demonstrated that it was not the complexity of the required movements (i.e., rotations in addition to translations) that made the task more difficult in the latter situation. Their study demonstrated that children's planning was primarily directed at attaining a toolto-target relation and not at these movement sequences per se. This result agrees with recent findings from imitation studies that show that children's imitation is not guided by the movement they see demonstrated to attain a goal, but by the goal they see realized (e.g., Bekkering, Wöhlslager, \& Gattis, 2000).

Finally, anthropological studies of tool use in hunter-gatherer cultures also stress the significance of the action-selection process. In our modern Western society, we are used to a plethora of artifacts, which are designed in such a way that it 
highly constrains possible actor-to-tool and tool-to-target relations and thus the variety of tool functions becomes severely limited. However, throughout the history of mankind tools were multipurpose devices. Only recently have they become specialized in their use and functions. For example, simple-looking tools (at least to modern Western eyes) like sticks were originally used for a wide range of functions, such as grasping, digging, pounding, making music, and communicating over large distances (Ingold, 1997). These different functions involved differences in the tool-to-target relation (e.g., type of grasp or grip position) and, accordingly, also in the actor-to-tool relation (e.g., point of contact or type of tooling action). For this the tool user had to choose which relation was demanded by which situation. Means-ends insight, therefore, involved the flexibility of selecting the appropriate relation from the various alternatives that were available for the task at hand. Studies by Roux, Bril, and colleagues (Roux \& Bril, 2005) on stone knapping in India demonstrated that highly skilled craftsmanship depends more on the flexibility with which different relations among stone, hammer, and anvil can be realized by the craftsman than on the ability to plan a large part of the activities ahead of time.

\section{PERSEVERATION IN TOOL USE: THE AIM OF THIS STUDY}

The study of perseveration in tool use may provide a window for understanding the dynamics of the choice process that underlies a child's means-ends insight. The problem of choosing among different tool-to-target relations and one relation dominating the other in the choice process might have led to results as reported by Van Leeuwen et al. (1994). In tasks such as theirs, several tool-to-target relations are feasible that can be divided in two categories: hook use and stick use. In stick use, the object can touch the tool at any position. In hook use, the target has to be located inside the hook. As the researchers suggested, the tool-to-target relation that dominated children's choices could have been stick use. To better understand why children persevered in using the cane in one way while ignoring to use it the other way, we have to investigate the many influences that affect children's choices.

The occurrence of perseverative behavior implies (at least) the following: (a) The presence of behavioral alternatives that are in the repertoire of the child. The child may attend to and even pick up the new task information, but for some reason this information does not "overrule" prior experiences at the moment the decision is made. (b) The presence of influences that are unsuccessful at affecting the choice that is made between these alternatives. Perservation means that a child "sticks" to previous choices while faced with a change in task setting that demands an alteration of this earlier choice. It is the state of prior choices in the action selection process that may prevent the child from making the switch. 
This study is directed at perseveration in young children's action selection when using a tool in a goal-directed task. In particular, this study addresses perseveration in the tool-to-target relation (Experiment 1) and the actor-to-tool relation (Experiment 2), when children use a tool for displacing an object to a goal position. In Experiment 1 they had to use a cane, which involved the choice of cane-to-object configuration. In Experiment 2 they had to use a spoon, which involved a choice of hand. We were interested in two questions: First, do children (spontaneously) choose the appropriate or adequate parameter setting on the first trial? Are they at least easily trainable, so that they do so in a reliable way afterward? This proves that they are able to filter the relevant information that enables them to perform the task, and that the possible alternatives are indeed in the child's behavioral repertoire. (If not, it would be insensible to ask the following question.) Second, when they have performed the task in one particular way a number of times in a row, will they persevere in doing so when task conditions change and another way to perform the task would be more appropriate? That is, do children adapt their behavior to the new task demands, or do these changed task demands fail to influence their choice of topology or hand, respectively? The experiments were performed with 3-year-old children, because at that age children's dexterity in using canes and spoons is variable enough to expect that they are able to perform these tasks.

\section{EXPERIMENT 1}

The first experiment investigated how young children's choices of how to use a tool came about in the context of a goal-directed task. The task involved repeated displacements of an object toward a goal position by using a cane. Children had to bring the object to a goal location in front of them, either at the near end of the table (proximal) or at the far end of the table (distal). We hypothesized that children's understanding of how to use the cane is not only determined by the position of the goal location relative to the object and the child, but also by the preference for a particular type of usage that has been set up during the task. Because of the rectangular shape of the cane's hook, it afforded pulling for moving objects nearby and pushing for displacing objects further away (see Figure 1). Pulling and pushing involve different tool-to-target relations (topologies; see Smitsman et al., 2005). In pulling, the topology is such that the object is located inside the hook, whereas for pushing the object is located outside the hook.

For this experiment, our research questions translated to the following: First, when children have to pull an object nearby or when they have to push the object further away, will they (spontaneously) choose enclosure within the hook as the topology in the former case and exclosure outside of the hook in the latter? Second, when children have initially pulled the object nearby repeatedly (i.e., a couple of 

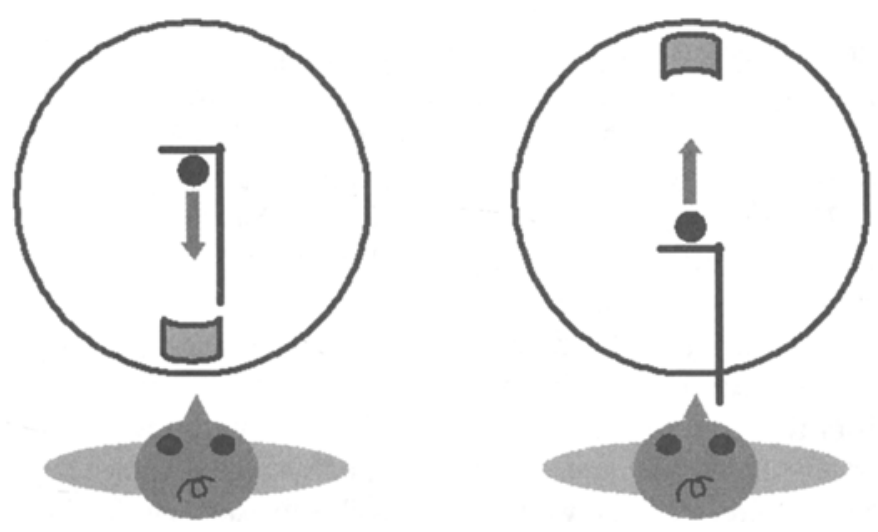

FIGURE 1 Setup of Experiment I: (a) An example of a trial with the proximal goal location. Also shown is an enclosure topology. (b) An example of a trial with the distal goal location. Also shown is an exclosure topology.

times in a row) by means of the enclosure topology, will they persevere in this topology when the goal location is moved so that it requires pushing? Or, will they immediately switch to the other (new) topology that is more appropriate for pushing, namely exclosure? The same question, of course, applies to the situation of first pushing and then pulling.

\section{Method}

Participants. Forty-three 3 -year-old children ( 15 boys and 28 girls) participated in the experiment. All children were within a 4-week range around their third birthday. The children were recruited by means of written invitations. Names and addresses of potential recruits were obtained through the birth records of the city of Nijmegen. The parents voluntarily replied with a return card, after which an appointment was made in a follow-up telephone call. The children and their parent(s) were rewarded for their participation by means of a certificate, photograph, and a small financial compensation.

Materials. Children performed the task on a height-adjustable table. The tabletop was round with a diameter of $66 \mathrm{~cm}$ (see Figure 1). A blue, curved pit of 7.0 $\mathrm{cm}$ by $12.0 \mathrm{~cm}$ and $2.6 \mathrm{~cm}$ deep, with its center at a distance of $7.5 \mathrm{~cm}$ from the edge of the tabletop, served as the goal location. The goal location was placed on the child's midline, either at the far end of the table or the near end of the table where the child was standing. We constructed a blue, hollow, aluminum tube (12 $\mathrm{mm}$ in diameter) into a cane, by bending one end to an angle of $90^{\circ}$ with respect to 
the handle. The cane was $38 \mathrm{~cm}$ long and had a mass of $36 \mathrm{~g}$. This cane served as the tool for displacing objects toward the goal location. As target objects we used plastic sea animals (one yellow and one green fish, and one orange octopus), about $5 \mathrm{~cm}$ (length) by $4 \mathrm{~cm}$ (width) by $4 \mathrm{~cm}$ (height) in size.

Procedure. The child was positioned in front of the table, with the height of the tabletop adjusted to the child's waist height. During task performance, children were standing on a footboard, which prevented them from walking around the table. If necessary, a parent was asked to keep the child in position by holding him or her at the waist or taking him or her between the knees. The parent was instructed not to hinder or help the child during the task, and to only give verbal encouragement without any hints.

Before the task started, the experimenter showed the cane and the target objects to the child, and allowed the child to explore them for a while. Furthermore, the experimenter pointed to the blue pit in the table and told the child that this was a water basin for the sea animals. The animals were too small to go there alone, and the child was asked to help them. They were encouraged to move the animals to the basin, with the cane. Then, the experiment started by placing one of the plastic animals near the middle of the tabletop at a clear distance from the basin. The stick was given to the child. The goal location varied, depending on the condition and the trial number (see later).

There were two conditions in the experiment to which children were assigned randomly. Each condition consisted of six trials: In one condition the child started with the goal location at the distal end of the table. This was maintained for a total of four subsequent trials. After this, the basin was rotated to the proximal end of the table. Two more trials followed with this goal location. In the other condition the order of the goal locations was reversed (i.e., proximal distal). In both conditions the order of the trials was according to the following schema: $A_{1}, A_{2}, A_{3}, A_{4}$, $B_{1}, B_{2}$. In this schema, the $A$ trials denote the initial goal location that was presented to the child (either proximal or distal); the $B$ trials denote the goal location after the switch (distal or proximal, respectively). Trials $A_{1}$ and $A_{2}$ also put children at ease with the task.

Response scoring. Digital video recordings were made of the experiments. From these recordings, task performance was scored in terms of one behavioral variable, topology, which reflected the orientation of the cane with respect to the object. The value this variable could attain was either enclosure or exclosure, which were the only two response categories for scoring (two-alternative forcedchoice). See Figure 1 for a typical example of both types of topologies. Every object-to-cane configuration where the object was touching the internal part of the $90^{\circ}$ hook-handle angle was scored as enclosure. Every object-to-cane configura- 
tion where the object was touching the external part, including the tip of the hook, was scored as exclosure.

One observer scored all trials and another observer scored a random sample of about one-quarter of the trials. Percentage of agreement between the observers was very high $(>94 \%$, Cohen's $\kappa=.86)$.

\section{Results and Discussion}

The percentages of use of the enclosure topology in the last two A trials and the two B trials are shown in Figure 2. ${ }^{1}$ From Figure 2, a number of things become apparent. First, children had a clear preference for enclosure. Overall, only $34 \%$ of the last two trials were performed with an exclosure topology, whereas $66 \%$ were performed with an enclosure topology. Even on the A trials of the distal-first condition, $38 \%\left(\mathrm{~A}_{3}\right)$ and $31 \%\left(\mathrm{~A}_{4}\right)$ of the children spontaneously transported the object by enclosing it within the hook of the cane. Nonetheless, the presence of both kinds of topologies in the first trials clearly demonstrates that both enclosure and exclosure were indeed realistic behavioral alternatives for the children.

Second, changes are visible in the percentages of enclosure and exclosure use between the A trials and B trials for both conditions as a consequence of experience with the other topology on the A trials (see Figure 2). After prior experience with the distal goal and pushing, the choice of the enclosure topology for the proximal goal and pulling was $56 \%$ (both $\mathrm{B}$ trials) compared to $100 \%\left(\mathrm{~A}_{3}\right)$ and $93 \%$ $\left(\mathrm{A}_{4}\right)$ without such experience. After prior experience with the proximal goal and pulling, the choice of the exclosure topology for the distal goal and pulling was $19 \%\left(B_{1}\right)$ and $26 \%\left(B_{2}\right)$ compared to $62 \%\left(A_{3}\right)$ and $69 \%\left(A_{4}\right)$ without such experience. This relatively large drop that occurred for both topologies clearly shows the influence of prior experiences on the topology children choose, in addition to task information.

Comparison of enclosure use on the $A_{3}$ trial of the condition (A proximal, $B$ distal) with that on the first B trial of the condition (A distal, B proximal) enables us to measure the effect of previous experience with a topology on subsequent choices for another topology. In other words, of the children who (spontaneously) used enclosure for pulling the object nearby on the first trials of the proximal-first condition, how many of them still used enclosure during the first proximal trial after a sequence of four distal trials? A similar comparison can be made for exclosure. Mann-Whitney $U$ tests were performed on the two topology distributions, for both possible comparisons (see Figure 2):

\footnotetext{
'Although children's choice of topology varied slightly over the four A trials, we used Trial $\mathrm{A}_{3}$ for comparison instead of Trial $A_{1}$ or $A_{2}$, to avoid possible effect of unfamiliarity and unease with the task.
} 


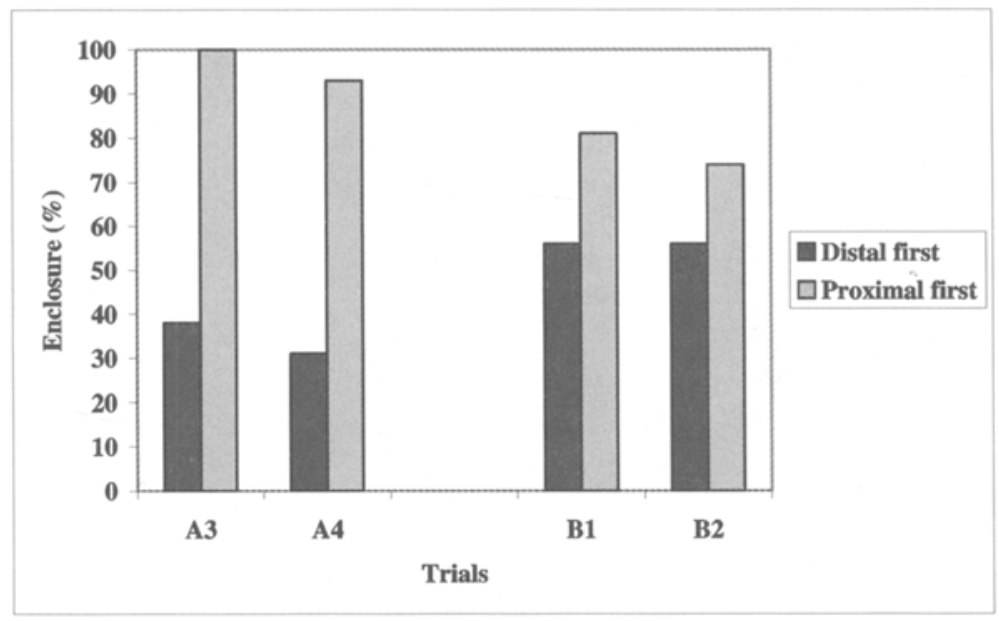

A

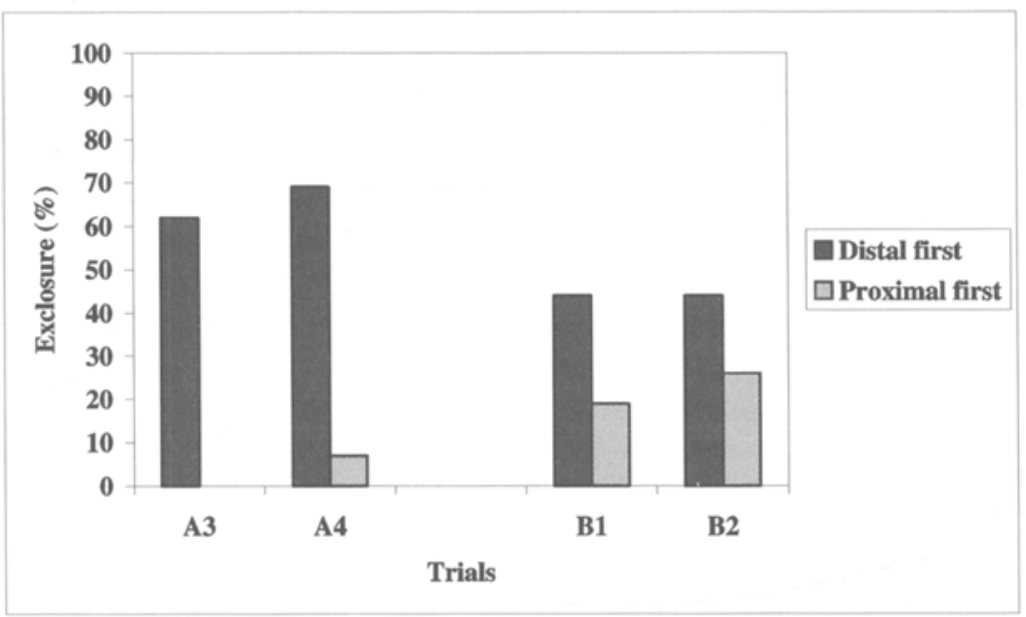

B

FIGURE 2 Graphic representation of the results of Experiment 1: Bars represent percentage of (a) enclosure and (b) exclosure topology choices in the A trials (without prior experience with the other topology) and the B trials (with prior experience with the other topology; i.e., after a switch of goal-location from distal to proximal or vice versa). Comparison of the height of the red bar on Trial A3 with the height of the blue bar on Trial B1 shows the amount of perseveration for (a) enclosure and (b) exclosure. The percentage of enclosure choices for a proximal target location on $B$ trials decreases as a result of prior experience with the distal target location (Figure 2a). The complementary percentage of exclosure choices for a proximal location on $\mathrm{B}$ trials increases as a result of prior experience with the distal target location (Figure $2 \mathrm{~b}$ ). 
1. Enclosure: Trial $A_{3}$ of $A$ proximal, $B$ distal condition with Trial $B_{1}$ of $A$ distal, B proximal condition.

2. Exclosure: Trial $A_{3}$ of $A$ distal, $B$ proximal condition with Trial $B_{1}$ of $A$ proximal, B distal condition.

The analyses demonstrate that both differences were significant, (Mann-Whitney $\mathrm{U}$ tests; $p s<.001$ ). Prior experience with a topology significantly reduced selection of another topology, which was demanded by the task switch, and which children would have selected without the prior experience. Summarizing, we must conclude that the kind of topology children selected depended not only on the constraints, in particular the goal location, but also on their earlier experiences during the task. Although children appeared to know which topology was most appropriate for which goal, they often selected an inappropriate one as a result of the experience with that particular topology during the preceding trials.

Finally, because children were not allowed to move around the table, an inappropriate choice of topology forced them to attain an awkward posture for displacing the object to its goal when they proceeded with the inappropriate topology. For instance, transporting the object to the distal (proximal) goal location using enclosure (exclosure) required the child to bend over the table very far, hold the cane at the handle very near to the hook, and turn the cane slightly such that the handle was not parallel to the movement path (Figure 1). These awkward postures and associated arm movements were not exceptional, yet they have indeed been regularly observed. In all cases, children mobilized these movements and postures to realize and maintain a specific tool-to-target relation.

\section{EXPERIMENT 2}

In the second experiment, hand choice was studied in young children's grasping of a spoon for performing a goal-directed task. As in Experiment 1, the task involved a repeated action after which a switch in the task conditions demanded children to adapt their behavior. The question was this: Will they change their behavior according to the new task demands or persevere? In separate series, children repeatedly fetched a spoon that was placed and oriented in such a way that it was either most easily grasped with the right hand or with the left hand. The switch after each of these series was to a neutral situation, where the spoon was on midline with the handle toward the child's belly. This setup enabled us to study whether hand choice (i.e., the actor-to-tool relation) was influenced by the previous experience of using a particular hand. 


\section{Method}

Participants. Twenty-six 3-year-old children (11 boys and 15 girls) participated in the experiment, all within a 4-week range around their third birthday. Recruitment and rewarding of participants was the same as in Experiment 1.

Material. A metal spoon, a part-wooden and part-plastic spoon holder, plastic toy paprika, and a terrycloth hand puppet were used (see Figure 3). The spoon had a handle of about $13 \mathrm{~cm}$ long. Its bowl was about $5.5 \mathrm{~cm} \times 4.5 \mathrm{~cm}$ and $1.5 \mathrm{~cm}$ deep, which minimized control demands during transportation. The mass of the spoon with the toy paprika was $56 \mathrm{~g}$, and the mass of the spoon alone was $37 \mathrm{~g}$. For easy grasping, the spoon was placed on the spoon holder about $10 \mathrm{~cm}$ above the table. The experimenter could open and close the hand puppet's mouth to play a "pretend feed" game.

Procedure. The task was performed at a regular table, with the child standing on a footboard at one side and an experimenter sitting at the other side. The child was allowed to explore all the objects, while the experimenter explained that the hand puppet needed to be fed with the spoon. Following this, the first trial of the experiment started, with the puppet on the experimenter's hand and the spoon with paprika on the holder, all out of reach for the child. The spoon was presented either in front of the child's left shoulder with the handle pointing to the left, or in front of the child's right shoulder with the handle pointing to the right. After a short delay, spoon plus holder were pushed toward the child in a straight line. When the spoon was within reach, the child was allowed to grasp it and feed the paprika to the hand puppet, which was always presented on the child's midline. After feeding, the spoon was retrieved and a new trial was started.

As in Experiment 1, two conditions of six trials each were performed. The first four trials in each condition provoked children to use their left hand or right
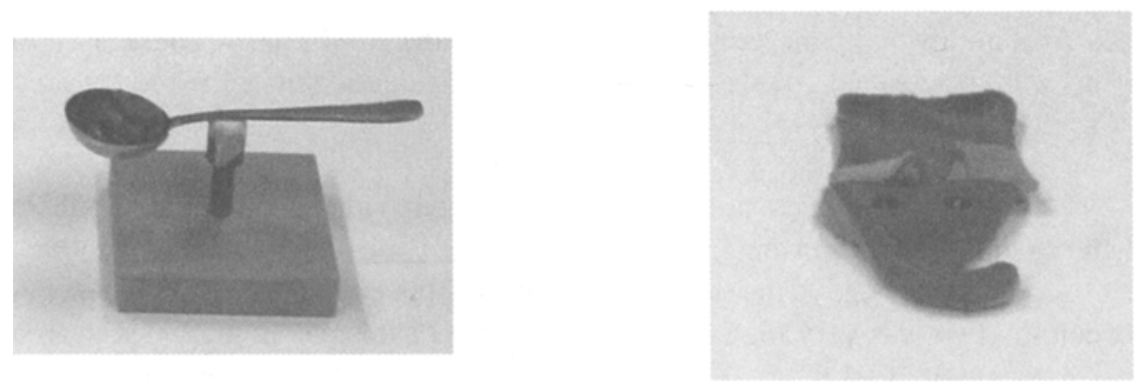

FIGURE 3 Materials used in Experiment 2: (a) The spoon with paprika placed on the spoon holder. (b) The terrycloth hand puppet, which could open and close its mouth by movements of the experimenter's hand. 

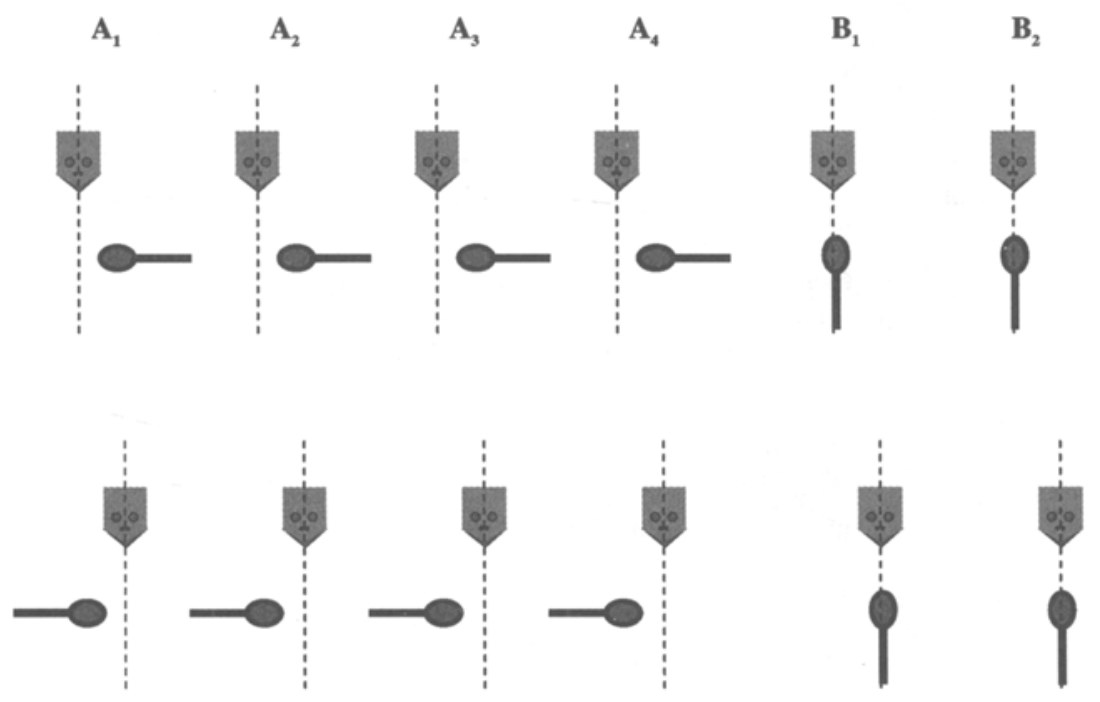

FIGURE 4 Schematic presentation of the trials sequences in the two different conditions of Experiment 2. Shown are the four A trials and the two B trials for both these conditions. The spoon is presented four times in front of the right hand (top), or four times in front of the left hand (bottom), before being presented two more times on midline.

hand for a number of times in a row. For this, the spoon was presented either four times on their left side with the handle pointing to the left or four times on their right side with the handle pointing to the right, respectively (see Figure 4). In the first two of these trials $\left(A_{1}\right.$ and $\left.A_{2}\right)$ the experimenter tried to "persuade" the child to use the appropriate hand by presenting the spoon somewhat more peripherally, if necessary. This was not done anymore in the third and fourth trials $\left(A_{3}\right.$ and $\left.A_{4}\right)$. The subsequent two trials $\left(B_{1}\right.$ and $\left.B_{2}\right)$ were laterally neutral in that they presented no bias toward using one of the hands. In the B trials, the spoon was presented at midline with the handle pointing toward the child's belly. These last two trials were performed to measure children's limb selection after the four biasing A trials.

Response scoring. An observer scored from videotaped sessions which hand a child used for grasping the object. This was compared to the judgment that was scored live by one of the experimenters during the experiment. Agreement between the two was very high ( $>96 \%$ ). In a case of mismatch, the video-scored value was used at all times. The possible values for hand use were left, right, or both. The latter value was assigned when both hands grasped the spoon at the same time. In the case of hand switching, the hand that grasped the spoon first was scored. 
TABLE 1

Results of Experiment 2: Percentage of Preferred-Hand Use in the Two Conditions

\begin{tabular}{lrrrrrr}
\hline Condition & \multicolumn{1}{c}{$A_{I}$} & \multicolumn{1}{c}{$A_{2}$} & \multicolumn{1}{c}{$A_{3}$} & \multicolumn{1}{c}{$A_{4}$} & $B_{I}$ & $B_{2}$ \\
\hline Spoon on PH side first & $100 \%$ & $100 \%$ & $100 \%$ & $100 \%$ & $96 \%$ & $85 \%$ \\
Spoon on NPH side first & $0 \%$ & $0 \%$ & $0 \%$ & $0 \%$ & $65 \%$ & $72 \%$ \\
\hline
\end{tabular}

Note. The A trials involve a lateral presentation of the spoon, either on the preferred-hand $(\mathrm{PH})$ side or on the non-preferred-hand (NPH) side. The B trials involve a presentation of the spoon on midline.

\section{Results}

The data from the right-hand condition and left-hand condition were transformed into a measure of performance based on each child's handedness: ${ }^{2}$ preferred-hand (PH) condition and non-preferred-hand (NPH) condition. In the PH condition the A trials consisted of spoon presentations on the child's PH side, whereas in the NPH condition the A trials consisted of spoon presentations on the child's NPH side.

In Table 1 the proportions of $\mathrm{PH}$ use are presented in the $\mathrm{A}$ trials and $\mathrm{B}$ trials of the two conditions. All children could be "trained" perfectly, which means that they performed no PH grasps in the A trials of the NPH condition, just as there were only PH grasps in the A trials of the PH condition. As can be seen in Table 1, after the switch from the A trials to the B trials, the amount of $\mathrm{PH}$ use in the $B$ trials differed between the two conditions. Because of the dichotomous repeated-measure data, McNemar tests were performed to analyze effects. These tests revealed that the differences in the proportion of $\mathrm{PH}$ use in Trial $\mathrm{B}_{1}$ between the two conditions were significant (McNemar test; $p<.01$ ). On the first $B$ trial of both conditions, a significant number of children persevered in using the same hand that they had used during the preceding A trials. For example, $65 \%$ of the children switched from their NPH to their PH, but only $4 \%$ switched from their PH to their NPH. This effect can only be caused by a (temporary) bias for using one of the hands, which has been built over these A trials. This difference between conditions was not significant for Trial $\mathrm{B}_{2}$ (McNemar test; $p>.5$ ).

\footnotetext{
${ }^{2}$ Children's individual handedness was determined before the start of the experiment by presenting the spoon three times on midline, with the handle pointing toward the child. In addition to this, a cylindrical object was also presented on midline several times. Children were asked to grasp these objects. A child was assigned to a particular handedness category (i.e., right-handed or left-handed) in correspondence with the hand that was used to make the largest number of grasps.
} 


\section{GENERAL DISCUSSION}

This study investigated 3-year-old children's action selection when they have to use a tool. In particular, the study addressed children's choices about the parameter setting in handling the tool with respect to two interconnected relations: the tool-to-target relation (configuration) and the actor-to-tool relation. The three questions we investigated are as follows: (a) Which parameters do children opt for on the first trials? (b) Does perseverative behavior occur when the task switches after a series of trials? (c) What are the multiple influences and time scales that affect decisions about the parameter setting as revealed by perseverative tendencies?

Experiment 1 investigated children's choices with respect to the tool-to-target relation when they had to move a toy, either inside the hook of a cane for pulling or outside the hook of the cane for pushing. Experiment 2 investigated children's choices with respect to the actor-to-tool relation, when they had to pick up a spoon with one hand for feeding a puppet. The spoon's position and orientation varied, and therefore afforded either a right-hand choice or a left-hand choice depending on whether the spoon was placed in the child's right hemispace with the handle pointing to the right, or in the left hemispace with the handle pointing to the left. Perseveration was subsequently studied by placing the spoon at midline with the handle pointing to the child's belly, after a series of lateral trials.

Results showed that both task information and accumulated experiences on earlier trials added to the action selection children made, in addition to their hand preference in Experiment 2. They initially selected the appropriate configuration in Experiment 1 and the "appropriate" hand (requiring least effort) in Experiment 2 , when they performed the task on the first trials. However after a number of trials with one particular configuration and hand, children persevered when the task required them to switch, irrespective of the configuration or hand they first used.

Perseveration was found in both experiments in a considerable number of children, although the task differed with respect to which tool had to be used (hook vs. spoon), which type of action was required (transporting vs. feeding), and which relation children had to control (tool-to-target vs. actor-to-tool). The occurrence of perseveration across different tasks, actions, and tools adds to the robustness of the phenomenon, underscoring Thelen et al.'s (2001) insight that it is neither task nor age bounded. This in turn adds to the view about the multiple determinedness and dynamics of the action-selection process. Influences at different time scales combine to affect choices made to organize the action.

What do our results add to other studies that also showed perseverative tendencies? Perseveration is not a rare phenomenon in young children's action selection as discussed in the introduction. Aguiar and Baillargeon (2000) showed perseveration in a cloth-pulling task in 6.5- and 9-month-old infants. Infants, who had to select which one of two cloths to pull for retrieving a toy, chose the cloth on which a toy was placed rather than the other cloth behind which a similar toy was 
placed. However they persevered in their earlier choice after switching the cloths; that is, when the cloth they pulled before now had the toy standing behind it and the other cloth now had the toy standing on it. Want and Harris (2001) found perseveration in a trap-tube task in 2-year-old and 3-year-old children. In their study, children had to retrieve a toy from a tube with a trap on one end by means of a stick. To push the object out, they needed to choose the correct end of the tube in which to insert the stick. Inserting the stick at the wrong end would push the toy into the trap, on its way out. Most 2-year-old and 3-year-old children (partially) succeeded in getting the toy out of the tube after a demonstration. However, when the tube was rotated between each trial, so that the "correct" end switched from side to side, nearly all 2-year-olds and a majority of the 3-year-olds persevered in their earlier choice. Want and Harris attribute the occurrence of perseveration and the inability to profit from a demonstration, to a lack of understanding of the causal structure of the task. Finally, perseveration also occurs in cart-sorting tasks, when the task requires children to switch between sorting dimensions, for instance, from color to shape or vice versa (Brace, Morton, \& Munakata, 2006; Zelazo, Frye, \& Rapus, 1996).

This study differs in two important respects from the studies mentioned earlier. First, it demonstrates perseveration of children's choices with respect to the principal relations that transform a handheld object into an effective means to attain a goal, and thus form the core of means-ends insight in tool use. In the cloth-pulling setup of Aguiar and Baillargeon (2000) the topology for pulling a toy nearby was fixed, or given by the display of the task. In card-sorting tasks, the experimenter tells the child the dimension he or she needs to attend to. In the trap-tube task, the trap that the child needs to avoid is also fixed. Although in our study the relations were also afforded within the goals and means presented in the task, they were not fixed. To realize these affordances children had to choose (a priori) among many different alternatives that were afforded. For instance, the cane afforded enclosure (i.e., pulling) as well as exclosure (i.e., pushing). The spoon (on midline) afforded a right-hand grasp as well as a left-hand grasp. The relations needed to be established by the children themselves by selecting a particular way to handle the tool from among the several possibilities. Second, the setup of the task and results revealed that several influences compete with each other. Jointly they determined which alternative prevailed in the choice process, which could be the correct or wrong one, depending on the joint effect of these influences. Together these aspects suggest that means-ends insight might emerge from the selection process, rather than being the cause of action as Want and Harris (2001) suggested.

Our point is that understanding how means and ends connect in an act cannot be conceived as existing independently from the process of making decisions regarding the act, and the multiple influences that affect those decisions. One cannot conclude what children do and do not understand without first taking into account the action-selection process. Insight emerges from the multiple influences that come 
together from moment to moment during task performance. If insight were to exist as the cause of action choices, we would have a hard time explaining why children also make incorrect choices while previously demonstrating "understanding" of what needs to be done. Other sources of information, in particular, prior experiences with the task, also influenced children to decide to use the inappropriate means. Despite their access and attention to the relevant task information, insight into what to do turned out to be fragile and easily perturbed, and in fact nonexistent outside the context of the task.

Learning about goals, means, and their interconnection forms an important engagement for young children on their developmental paths for becoming generally skilled performers for a wide range of everyday tasks. Learning of affordances can also be conceived of in this way, as well as learning of action-effect relations from a slightly different theoretical stance (Bushnell et al., 2005; Hommel, 2003, 2006). In the process of discovering relations between means and ends, children actively explore the parameter settings that connect the two. Results of this study indicate that investigating and discovering action-effect relations is mediated by the selection of what to do and what not to do. Means that are available among the environmental objects (i.e., tools) are explored, including the degrees of freedom that are free to regulate when these tool becomes part of the action system (Smitsman, 1997).

The important question, then, is how does this learning take place when multiple influences at different time scales determine what children attend to, what they remember, and which choices they make to organize the action. The presence of multiple influences indicates that the learning may be more capricious than the formation of simple associations between causes and effects. Deeper rooted preferences from the motor system, such as the hand preference; shorter living influences, such as earlier experiences with a task; and online task information may affect children's choices and as a consequence the effect that occurs. They may persevere although they attended to changing task information that demanded a different choice. On the other hand the combined effect of these multiple influences in the intrinsic dynamics of the selection process (beyond the focus of this study), may also facilitate the discovery of relevant parameters and, as a consequence, speed up learning. This is illustrated by the observation we made when children incorrectly opted for enclosure to push a toy further away or for exclosure to pull a toy nearby. To accomplish the task, they often did not switch to the other more useful topology; instead they organized the degrees of freedom of the body differently, leading to awkward arm and body postures. Another example is 3-year-olds' perseverative behavior in a cart-sorting task (Brace et al., 2006). Intermediate practice with sorting cards that differed in only one dimension (e.g., color) was sufficient to facilitate switching from sorting on the basis of shape to sorting with respect to color. Without such experience, children persevered when they were instructed to switch dimension. 
Finally, Thelen persuasively advocated a complex systems view on the evolution of children's insight in what to do. According to her, insight emerges in situated and embodied organisms when motor, perceptual, and cognitive influences cooperate in the selection of where to look, reach, and what to pay attention to. This research and other research of ours (Cox \& Smitsman, 2007a, 2007b) supports and extends her view by showing the simultaneous presence of perceptual, cognitive (memory) as well as motor influences on the decisions children and adults make in various tasks. It also reveals that her view can be extended to the domain of tool use and means-ends insight. As we argued before, means-ends insight rests on the confluence of several sources of information in the action-selection process. In tool use the situation is not fundamentally different from tasks Thelen studied, in particular, the A-not-B task. It is more complicated because a tool object puts additional and adjusted constraints on the action system to transform the body and tool system into an effective goal-directed device. The fact that we discovered similar phenomena and processes in these more complicated tasks argues for the depth and breadth of Thelen's pioneering insights.

\section{REFERENCES}

Aguiar, A., \& Baillargeon, R. (2000). Perseveration and problem solving in infancy. In H. Reese (Ed.), Advances in child development and behavior (Vol. 27, 135-180). New York: Academic.

Baillargeon, R. (1993). The object concept revisited: New directions in the investigation of infants' physical knowledge. In C. E. Granrud (Ed.), Visual perception and cognition in infancy (pp. 265-315). Hillsdale, NJ: Lawrence Erlbaum Associates, Inc.

Baillargeon, R. (1994). How do infants learn about the physical world? Current Directions in Psychological Science, 3, 133-140.

Baillargeon, R. (1998). Infants' understanding of the physical world. In M. Sabourin \& F. Craik (Eds.), Advances in Psychological Science, Vol 2: Biological and Cognitive Aspects. Hove, England: Psychology Press/Erlbaum.

Bates, E., Carlson-Luden, V., \& Bretherton, I. (1980). Perceptual aspects of tool-using in infancy. Infant Behavior and Development, 3, 181-190.

Bekkering, H., Wohlschläger, A., \& Gattis, M. (2000). Imitation of gestures in children is goal-directed. Quarterly Journal of Experimental Psychology, 53A(1), 153-164.

Boudreau, J. P., \& Bushnell, E. W. (2000). Spilling thoughts: Configuring attentional resources in infants' goal-directed actions. Infant Behavior and Development, 23, 543-566.

Brace, J. J., Morton, J. B., \& Munakata, Y. (2006). When actions speak louder than words: Improving children's flexibility in a card sorting task. Psychological; Science, 17, 665-669.

Bushnell, E. W. (1985). The decline of visually guided reaching in infancy. Infant Behavior and Development, 63, 1142-1163.

Bushnell, E. W., \& Boudreau, J. P. (1998). Exploring and exploiting objects with the hands during infancy. In K. J. Connolly (Ed.), The psychobiology of the hand: Clinics in Developmental Medicine 147 (pp. 144-161). London: MacKeith Press.

Bushnell, E. W., Sidman, J., \& Brugger, A. (2005). Transfer according to the means in human infants: The secret to generative tool-use? In V. Roux \& B. Bril (Eds.), Stone knapping: The necessary condi- 
tions for a uniquely hominid behavior (pp. 303-317). Cambridge, UK: McDonald Institute Monograph Series.

Cox, R. F. A., \& Smitsman, A. W. (2006a). Action planning in young children's tool use. Developmental Science, 9, 628-641.

Cox, R. F. A., \& Smitsman, A. W. (2006b). The planning of tool-to-object relations in young children. Developmental Psychobiology, 48, 178-186.

Cox, R. F. A., \& Smitsman, A. W. (2007a). Action-selection perseveration in young children: Advances of a dynamic model. Manuscript submitted for publication.

Cox, R. F. A., \& Smitsman, A. W. (2007b). A dynamic model for limb selection. Manuscript submitted for publication.

Gibson, E. J., \& Pick, A.D. (2000). An ecological approach to perceptual learning and development. Oxford, UK: Oxford University Press.

Gibson, J. J. (1986). The ecological approach to visual perception. Boston: Houghton-Mifflin. (Original work published 1979)

Goldfield, E. C. (1995). Emergent forms: Origins and early development of human action and perception. New York: Oxford University Press.

Hommel, B. (2003). Planning and Representing Intentional Action. The Scientific World Journal. 3, 593-608.

Hommel, B. (2006). How we do what we want: A neuro-cognitive perspective on human action planning. In R J. Jorna, W. van Wezel, \& A. Meystel (Eds.), Planning in Intelligent Systems: Aspects, motivations and methods (pp. 23-56). New York: Wiley \& Sons.

Ingold. T. (1997). Eight themes in the anthropology of technology. Social Analysis, I, 106-138.

Lockman, J. J. (2000). A perception-action perspective on tool use development. Child Development, $71,137-144$.

Lockman, J. J. (2005). Tool use from a perception-action perspective: Development and evolutionary considerations. In V. Roux \& B. Bril (Eds.), Stone knapping: The necessary conditions for a uniquely hominid behavior (pp. 319-330). Cambridge, UK: McDonald Institute Monograph Series.

Piaget, J. (1954). The construction of reality in the child. New York: Basic Books.

Roux, V., \& Bril, B. (2005). General introduction: A dynamic systems framework for studying a uniquely hominin innovation. In V. Roux \& B. Bril (Eds.), Stone kanpping: The necessary conditions for a uniquely hominid behavior (pp. 1-18). Cambridge, UK: McDonald Institute Monograph Series.

Schöner, G., \& Dineva, E. (2007). Dynamic instabilities as mechanisms for emergence. Developmental Science, $10(1), 69-74$.

Schöner, G., \& Thelen, E. (2006). Using dynamic field theory to rethink infant habituation. Psychological Review, 113, 273-299.

Schutte, A. R., \& Spencer, J. P. (2002). Generalizing the dynamic field theory of the A-not-B error beyond infancy: Three-year-olds' delay- and experience-dependent location memory biases. Child Development, $73,377-404$.

Schutte, A. R., Spencer, J. P., \& Schöner, G. (2003). Testing the dynamic field theory: Working memory for locations becomes more spatially precise over development. Child Development, 74, 1393-1417.

Smitsman, A.W. (1997). The development of tool use: Changing boundaries between organism and environment. In C. Dent-Read \& P. Zukow-Goldring (Eds.), Evolving explanations of development: Ecological approaches to organism-environment systems (pp. 301-309). Washington, DC: APA.

Smitsman, A. W., \& Bongers, R. M. (2003). Tool use and tool making: A dynamical developmental perspective. In J. Valsiner \& K. J. Connolly (Eds.), Handbook of developmental psychology (pp. 172-194). London: Sage.

Smitsman, A. W., Cox, R. F. A., \& Bongers, R. M. (2005). Action dynamics in tool use. In V. Roux \& B. Bril (Eds.), Stone knapping: The necessary conditions for a uniquely hominid behavior (pp. 129-147). Cambridge, UK: McDonald Institute Monograph Series. 
Stedron, J. M., Sahni, S. D., \& Munakata, Y. (2005). Common mechanisms for working memory and attention: The case of perseveration with visible solutions. Journal of Cognitive Neuroscience, I7, 623-631.

Thelen, E., Schöner, G., Scheier, C., \& Smith, L. B. (2001). The dynamics of embodiment: A field theory of infant perseverative reaching. Behavioral and Brain Sciences, 24, 1-86.

Thelen, E., \& Smith, L. B. (1994). A dynamic systems approach to the development of cognition and action. Cambridge, MA: MIT Press.

Thelen, E., \& Whitmeyer, V. (2005). Using dynamic field theory to conceptualize the interface of perception, cognition and action. In J. J. Rieser, J. J. Lockman, \& C. A. Nelson (Eds.), Action as an organizer of learning and development (pp. 243-280). Mahwah, NJ: Lawrence Erlbaum Associates, Inc.

Tomasello, M. (1999). The Cultural Origins of Human Cognition. Cambridge (MA): Harvard University Press.

van Leeuwen, L., Smitsman, A.W., \& van Leeuwen, C. (1944). Affordances, perceptual complexity, and the development of tool-use. Journal of Experimental Psychology: Human Perception and Performance, 20, 174-191.

Want, S. C., \& Harris, P. L. (2001). Learning from other people's mistakes: Causal understanding in learning to use a tool. Child Development, 72, 431-443.

Willats, P. (1999). Development of means-end behaviour in young infants: Pulling a support to retrieve a distant object. Developmental Psychology, 35, 651-657.

Zelazo, P. D., Frye, D., \& Rapus, T. (1996). An age-related dissociation between knowing rules and using them. Cognitive Development, 11, 37-63. 